\title{
Influence of quenched jets on di-hadron correlations
}

\author{
Jiangyong $\mathrm{Jia}^{1,2, \circledast}$ and Roy Lacey ${ }^{1}$ \\ ${ }^{1}$ Department of Chemistry, Stony Brook University, Stony Brook, NY 11794, USA \\ ${ }^{2}$ Physics Department, Brookhaven National Laboratory, Upton, NY 11796, USA
}

(Dated: November 13, 2018)

\begin{abstract}
A simple jet absorption model is used to study the influence of hadron pairs produced by quenched jets, on di-hadron angular correlations at intermediate transverse momentum $\left(p_{T}\right)$. We demonstrate that such pairs can dominate both the yield and the shape of angular correlations, and may account for the similar properties between the near-side "ridge" and the away-side "double-humped" structure seen in recent data. These hadron pairs also show azimuthal anisotropy which is sensitive to the emission angle of hadrons relative to that of the jet. Measurement of this anisotropy may provide a constraint for elucidating the production mechanism for near- and away-side hadron pairs at intermediate $p_{T}$.
\end{abstract}

PACS numbers: 25.75.-q

\section{INTRODUCTION}

A new state of nuclear matter has been created in central $\mathrm{Au}+\mathrm{Au}$ collisions $\left(\sqrt{s_{N N}}=200 \mathrm{GeV}\right)$ at the Relativistic Heavy-ion Collider. This matter behaves like a fluid of strongly interacting quarks and gluons as indicated by its strong collective flow, low kinetic shear viscosity, and large opacity [1]. It is now commonly referred to as the strongly coupled quark-gluon plasma (sQGP).

Energetic jets, through their absorption pattern in this medium, can be used to probe the sQGP. Such a pattern results from the large energy loss suffered by jets traversing the medium, which leads to a suppression of hadron yields at high $p_{T}$ [2], a phenomenon known as jet quenching. A small fraction of jets, especially those emitted from the surface of the interaction zone, do escape the medium. These survived jets dominate both the single hadron and the pair yields at high $p_{T}(>5 \mathrm{GeV} / c)$, and appear in correlation analyses as narrow peaks at $\Delta \phi \sim 0$ (near-side) and $\Delta \phi \sim \pi$ (away-side) $[3$, 4$]$.

A majority of jets are produced inside the collision zone and are therefore quenched. The energy and momentum of these quenched jets are redistributed in the medium and are eventually shared among soft hadrons. These "medium response" or "feedback" hadrons can maintain a good degree of angular correlation with the original jets [5], depending on the details of the energy loss mechanism and the energy dissipation process. Such residual correlations are believed to give rise to the awayside double-humped structure peaks at $\Delta \phi \sim \pi \pm 1.1$ (the cone) [6, 7] and the near-side elongation in $\Delta \eta$ (the ridge) [7, 10], observed at low $p_{T}(<4 \mathrm{GeV} / c)$.

In general, correlated hadrons can be chosen from either survived jets or quenched jets. Therefore, jetinduced pairs can be divided into three groups,

- jet-jet pair: both hadrons come from fragmentation

${ }^{*}$ Correspond to jjia@bnl.gov of survived jets.

- jet-medium pair: one hadron comes from fragmentation of survived jet, the second hadron comes from medium feedback of quenched jet.

- medium-medium pair: both hadrons come from medium feedback of quenched jets.

The three groups of pairs have very different geometric origin. For jet-jet pairs, surface emission dominate the production of both hadrons (e.g., tangential emission); for jet-medium pairs, surface emission and volume emission dominate the production of the jet and medium hadrons, respectively; for medium-medium pairs, volume emission dominates the production of both hadrons.

In many previous correlation analyses, it is normally assumed that one hadron ("trigger") comes from the jet, and the second hadron ("partner") comes from either fragmentation or feedback, i.e., only jet-jet and jetmedium pairs are considered. In this picture, the trigger comes from a jet close to the surface, which losses some energy and fragments outside the medium. The fragments contribute to the near-side jet peak, and the feedback of the lost energy gives rise to the near-side ridge. Meanwhile, the away-side jet is quenched as it traverses a longer medium, and becomes the away-side cone.

This picture does not include the contribution from medium-medium pairs, which do not suffer surface-bias. In a jet absorption picture, the jet fragmentation contribution is proportional to the number of survived jet, i.e., $\propto R_{A A}^{0}$ (the constant suppression level at large $p_{T}$ ), while the medium response is proportional to the number of quenched jets, i.e., $\propto 1-R_{A A}^{0}$. Therefore the yields of inter-jet pairs from jet-jet, jet-medium, and mediummedium should scale as $\left(R_{A A}^{0}\right)^{2}, R_{A A}^{0}\left(1-R_{A A}^{0}\right)$, and $\left(1-R_{A A}^{0}\right)^{2}$, respectively. In the limit of strong quenching, $R_{A A}^{0} \rightarrow 0$, the medium-medium pairs dominate.

Experimentally, an enhancement of hadron pairs from quenched jets is observed, and their spectra are found to be softer than those from survived jets [7]. Therefore, medium-medium pairs are expected to be important only 
at intermediate and low $p_{T}$. Indeed, the low $p_{T}$ autocorrelation data from the STAR Collaboration suggests that each particle has 2-3 correlated particles in cental $\mathrm{Au}+\mathrm{Au}$ events [11]. The modification factor for hadronpair yields, for $p_{T}^{A}+p_{T}^{B}<7 \mathrm{GeV} / c$, has been shown to exceed the jet fragmentation limit [7], further suggesting that feedback hadron pairs dominate over those from jet fragmentation for $p_{T}^{A}=p_{T}^{B} \sim 3.5 \mathrm{GeV} / c$. These results imply that each jet may induce more correlated hadrons via medium response than by fragmentation. Furthermore, a striking similarity between the $p_{T}$ slopes and the particle species composition of the ridge and the cone has been reported [7, 8, 9]. The respective $p_{T}$ ranges over which the yields of these hadrons are important are also the same $(<4 \mathrm{GeV} / c)$ [7]. These similarities suggest that mechanisms for the ridge and the cone could be related. They also suggests that the bulk physics, hydrodynamical flow and parton recombination, should play an important role in this $p_{T}$ region.

On the theory side, several models for the medium response to jets have been proposed [12]. Some involve new particle production mechanisms such as bremsstrahlung gluon radiation or Cerenkov gluon radiation. Others involve mechanisms related to local heating of medium by the jet, such as momentum kick, jet deflection, machcone, etc. The latter mechanisms are very effective at generating large yield of correlated hadron pairs. However, they have focused primarily on jet-jet and jetmedium pairs, and because of the different geometrical origins for near- and away-side pairs, the proposed mechanisms for the ridge and the cone are also quite different.

An important question is the degree to which mediummedium pairs serve to influence di-hadron correlations, and whether or not they can give new and unifying insights on the observed medium response for the nearand away-side jets. In this paper, we investigate the possible role of the correlations among feedback hadrons via a simple phenomenological jet absorption model. We do not attempt to provide an explanation of the mechanistic origin of the jet quenching and the medium response, but focus instead on how they influence the relative contribution of jet-jet, jet-medium and medium-medium pairs, and their dependence on the collision geometry.

\section{THE MODEL}

We start with the jet absorption picture of Ref. [13], which assumes that jets loose all energy after one interaction in the medium. The corresponding energy loss probability distribution is

$$
P(\Delta E)=f \delta(\Delta E)+(1-f) \delta(\Delta E-E)
$$

The survival probability $f=e^{-\kappa I}$ is controlled by absorption strength $\kappa$ and matter integral $I$. For a parton produced at $(x, y)$ in the transverse plane, moving along direction $\left(n_{x}, n_{y}\right)$, the integral can be expressed as

$$
I=\int_{0}^{\infty} d l l \frac{c \tau}{l+c \tau} \rho\left(x+(l+c \tau) n_{x}, y+(l+c \tau) n_{y}\right),
$$

which corresponds to a quadratic dependence of absorption $(\propto l d l)$ in a longitudinal expanding medium $(\propto c \tau /(c \tau+l))$ with a formation time of $\tau=0.2 \mathrm{fm} / c$.

The collision geometry is modeled by a Monte Carlo Glauber simulation of $\mathrm{Au}+\mathrm{Au}$ collisions with a WoodsSaxon distribution for the Au nucleus. We calculate the participant density profile $\rho_{\text {part }}(x, y)$ and the binary collision density profile $\rho_{\text {coll }}(x, y)$ in the transverse plane. Back-to-back dijet pairs are generated according to $\rho_{\text {coll }}$ with a randomly selected azimuthal direction. These pairs are then swum through the medium whose density is $\rho_{\text {part }}$. We vary $\kappa$ to reproduce the observed constant suppression factor of 4.4 for hadrons in $0-5 \%$ central collisions [14]. This gives the value $\kappa=0.7$ that is then used for all other centrality selections. This approach was shown to reproduce the centrality dependence of singleand di-hadron yield at high $p_{T}[13$. It decouples the details of the energy loss process encoded in absorption strength $\kappa$, from the geometry of the medium encoded in the integral $I$, and allows us to focus on the geometric aspect of the interactions between jet and medium.

The average multiplicity for a jet emitted from a given location with a given direction in the collision zone is

$$
\langle N\rangle=\left\langle N_{j e t}\right\rangle e^{-k I}+\left\langle N_{m e d}\right\rangle\left(1-e^{-k I}\right),
$$

where $N_{j e t}$ is the number of fragmentation hadrons for each survived jet and $N_{\text {med }}$ is the number of medium hadrons induced by each quenched jet. $N_{j e t}$ and $N_{\text {med }}$ are assumed to follow Poisson distributions with a mean of 1 and 2 , respectively. The value of $\left\langle N_{j e t}\right\rangle$ roughly corresponds to the number of hadrons in 1-3 GeV/c for $6 \mathrm{GeV} / c$ jet; the value of $\left\langle N_{m e d}\right\rangle$ is chosen to account for the factor of 2-3 enhancement in the per-trigger yield seen in central $\mathrm{Au}+\mathrm{Au}$ collisions. This particular choice of $\left\langle N_{\text {jet }}\right\rangle$ and $\left\langle N_{\text {med }}\right\rangle$ does not influence our conclusions as long as $\left\langle N_{\text {med }}\right\rangle>\left\langle N_{\text {jet }}\right\rangle$.

For each di-jet, the total number of hadrons, $N_{t o t}$, is the sum of the multiplicity of the two jets, $N_{1}$ and $N_{2}$. $\quad N_{1}$ (and $N_{2}$ ) equals either $N_{j e t}$ (if the jet survives the medium) or $N_{\text {med }}$ (if the jet is quenched). The total number of pairs induced by the di-jet is simply $N_{t o t}\left(N_{t o t}-1\right) / 2$. The azimuthal shape of these pairs is fixed by the kinematics for jet fragmentation and the medium response as follows. The distribution of jet hadrons is determined by the fragmentation process and initial parton transverse momentum $k_{T}$. The near-side and away-side widths are related to each other by $\sigma_{\text {away }}=\sqrt{\sigma_{\text {near }}^{2}+\sigma_{k_{T}}^{2}}$, where $\sigma_{k_{T}}$ is the smearing due to $k_{T}$. We chose $\sigma_{\text {near }}=0.2$ and $\sigma_{k_{T}}=0.4$ to match the measured widths for $1-3 \mathrm{GeV} / c$ range in $p+p$ and $d+A u$ collisions [15]. For medium hadrons associated with a quenched jet, we distribute them at $\pm(D=1.1)$ radian [7] (relative to the initial direction of the parton) 
with a Gaussian width of 0.3 radian. We also assume that only $30 \%$ of the inter-jet pairs are detected, so as to account for finite detector acceptance and the swing of the away-side jet in pseudo-rapidity. ${ }^{1}$

We note that for models where only the jet-jet and jet-medium pairs are included, this choice of $\mathrm{D}$ provides a good match for the away-side cone structure. In our simulation, we study medium response patterns where feedback hadrons are emitted equally on two sides of the original jet ("mach-cone" type) or one side of the original jet ("deflected-jet" type). These two patterns are indistinguishable for jet-medium pairs, but they do lead to different shapes for medium-medium pairs.

In di-hadron correlation analysis, one usually use pertrigger yield (PTY) and the corresponding modification factor $I_{A A}$, the ratio of per-trigger yield in $\mathrm{Au}+\mathrm{Au}$ collisions to that in $p+p$ collision, to characterize the jet quenching and medium response. PTY and $I_{A A}$ are good variables for high $p_{T}$ correlation, since trigger yield is dominated by jet fragmentation and each trigger tags one jet, such that per-trigger yield (PTY) is a good representation of per-jet yield. But at $p_{T}<4 \mathrm{GeV} / c$ region, nonfragmentation triggers from soft production mechanisms or medium response mechanisms become important. Experimental evidences include a particle composition that is strongly modified relative to that in $p+p$ collisions [16], a $R_{A A}$ and an elliptic flow that peak in this momentum region at a level much bigger than what one expect from the jet quenching [14, 17]. These soft triggers tend to dilute the $I_{A A}$, since they either have no correlation or non-jet like correlation. This is especially important for studying the dihadron as function of angle with respect to the reaction plane $(\mathrm{RP})$. Because the yield of soft triggers varies with the angle w.r.t the RP, thus the dilution effect to PTY also varies with the trigger direction.

Experimentally, such a dilution effect has been observed in a recent PHENIX analysis [7, 18]. As proposed in [7], an alternative observable for describing the medium response is the hadron pair yield (the total number of correlated pairs per event) and the corresponding modification factor $\left(J_{A A}\right)$ in $\mathrm{Au}+\mathrm{Au}$ collisions. For this reason, we present most of the results in term of pair variables, pair yield and pair $v_{2}$ (Eq. 4).

\section{RESULTS}

Figure1 compares the pair yields for jet-jet,jet-medium and medium-medium pairs as a function of centrality. The decrease with $N_{\text {part }}$ for jet-jet pairs is caused by the onset of jet quenching; the increase of medium-medium pairs signifies the onset of medium response. The nonmonotonic trend for jet-medium pairs reflects the compe-

\footnotetext{
1 The typical width of the away-side jet in $\eta$ is \pm 2 to \pm 3 , a detector covers \pm 0.7 in $\eta$ accepts about $30 \%$ inter-jet pairs.
}

tition between jet fragmentation and medium response. The ordering of the three sources in central collisions, medium-medium $>$ jet-medium $>$ jet-jet, is a natural consequence of $\left.\left\langle N_{\text {med }}\right\rangle\right\rangle\left\langle N_{\text {jet }}\right\rangle$. That is, each quenched jet gives rise to more pairs than a survived jet. It is a necessary condition required to explain the enhancement of the pair yields.

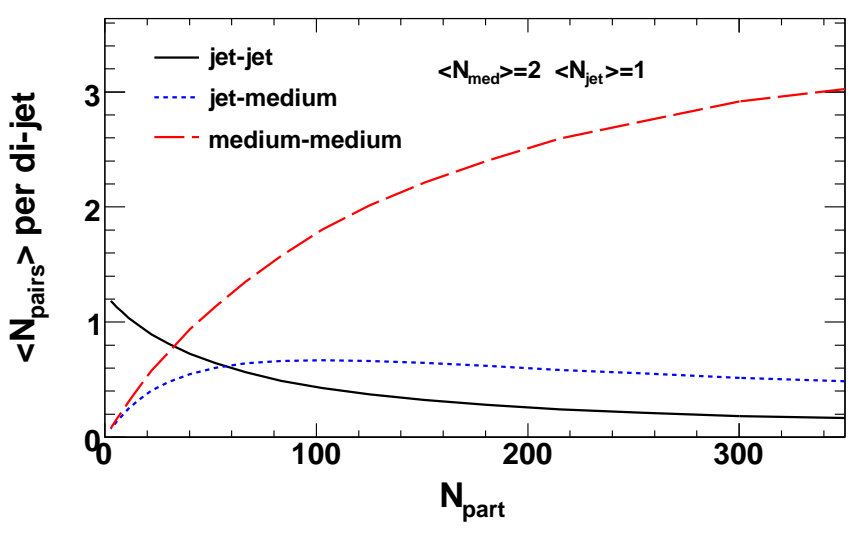

FIG. 1: (Color online) The centrality dependence of pair yield normalized by number of dijets from jet-jet, jet-medium and medium-medium sources.

Figure 2] shows the $\Delta \phi$ distribution for jet-jet and jet-medium pairs, which dominates the near-side and the away-side, respectively. There are no jet-medium pairs on the near-side, because a single jet in our simulation does not simultaneously produce feedback- and fragmentation-hadrons.

Figure 2r summarizes the medium-medium pairs distribution for the mach-cone scenario. The contributions for intra-jet pairs (referring to pairs from the same jet) and inter-jet pairs (referring to pairs where one hadron comes from one jet and the other comes from the opposite jet) are shown separately. The emission directions for hadrons contributing to medium-medium pairs are illustrated in Fig. 2a. In this picture, both jets are quenched and converted into hadrons at angle $\pm D$ radians relative to the original jet direction. The pairs constructed from these hadrons, when plotted in $\Delta \phi$, should concentrate at $\Delta \phi \sim 0, \pi, \pm 2 D$ and $\pi \pm 2 D$. The intra-jet pairs split up into three branches at $\Delta \phi \sim 0,2 D$ and $-2 D$, while the inter-jet pairs have a sizable peak at $\Delta \phi \sim \pi$ and two small satellite peaks around $\Delta \phi \sim \pi \pm 2 D$. Since the value of $D=1.1$ implies $2 D \approx \pi-D$, the pairs from the same jets (at $\pm 2 D$ ) coincide with the location of jetmedium pairs (at $\pi \pm D$ ). Fig. 2 d contrasts contributions from all three sources. We see that the medium-medium pairs alone already account for the double-humped structure on the away-side.

Figure $2 \mathrm{~d}$ shows that the medium-medium pairs dominate the intermediate $p_{T}$ correlation signal. Furthermore, Fig. 25 shows that most of these pairs, both in the near-side peak and in the away-side double-humps, originate from the same jet (intra-jet pairs). Because of 

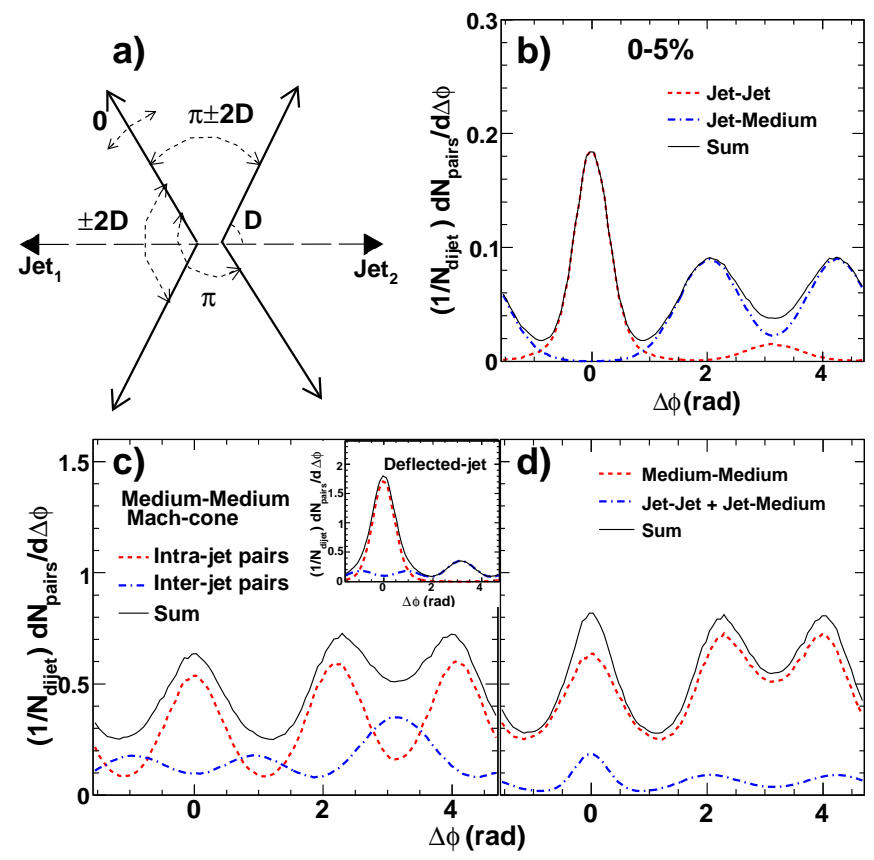

FIG. 2: (Color online) $\Delta \phi$ distributions in $0-5 \%$ central $\mathrm{Au}+\mathrm{Au}$ collisions for $\mathrm{b}$ ) jet-jet and jet-medium pairs, c) medium-medium pairs from mach-cone scenario (deflected-jet scenario is shown as insert), and d) comparison of jet-jet plus jet-medium with medium-medium from mach-cone scenario, normalized by the number of generated dijets. Panel a) illustrates the typical emission directions (solid arrows) and $\Delta \phi$ values (numbers) for medium-medium pairs.

this common origin, the near-side and away-side medium response pairs naturally should have the same properties, i.e., common particle composition, common slopes, etc. Now if there are some mechanisms which broaden the hadrons of a jet along the pseudo-rapidity, all intrajet medium-medium pairs from the jet should also be elongated in $\Delta \eta$. In this case, the experimentally observed ridge and cone which are flat in $\Delta \eta$ simply originate from the longitudinal broadening of those intra-jet medium-medium pairs that appear at the near-side and the away-side, respectively. ${ }^{2}$

The insert in Fig. 2r shows the medium-medium pair $\Delta \phi$ distribution for the deflected-jet scenario. It shows a large peak on the near-side, but the away-side does not exhibit the cone structure. This distribution is in stark contrast to the ones for jet-medium pairs in Fig. 2 $\mathrm{b}$, which is indistinguishable for the mach-cone and deflected-jet scenarios. In what follows, we limit our dis-

\footnotetext{
2 Note that all intra-jet pairs, appearing on both near- and awayside, should be elongated in $\Delta \eta$. It is relatively easy to identify the ridge on the near-side. However, it is harder to do so at the away-side because its net effect is indistinguishable from the jet swing which also results in an elongation along the beam direction.
}

cussion of medium-medium pairs to those from the machcone scenario.

Due to the asymmetry of the overlap region of the two nuclei, the matter integral $\mathrm{Eq} 2$ for a jet varies with its azimuthal direction with respect to the reaction plane. This leads to an azimuthal anisotropy of emitted pairs, characterized by pair $v_{2}$

$$
v_{2}^{\text {Pair }}=\frac{\int \cos 2\left(\phi-\Psi_{R P}\right) \text { PairYield }(\phi) \mathrm{d} \phi}{\int \operatorname{PairYield}(\phi) \mathrm{d} \phi}
$$

The $\phi$ in Eq4 can refer to either the angle of the original jet or the angle of one of the hadron from the pair. Motivated by earlier discussions, we use pair yield instead of per-trigger yield for studying the reaction plane dependence, because soft non-fragmentation triggers obscure the meaning of per-trigger yield, and the yield of these soft triggers varies with the angle with respect to the reaction plane.

The $v_{2}$ values calculated for jet-jet, jet-medium and medium-medium pairs are found to be different due to differences in their geometrical origin. Fig. 3 and Fig. 4 summarize the centrality dependence of the $v_{2}$ 's for the different pair types. The $v_{2}$ 's for jet-jet pairs are calculated using the $\phi$-angle of the parent jets as was done in Ref. 13] (solid lines) or by using the $\phi$-angle of fragmentation hadrons (dotted lines). We note that the $v_{2}$ 's for intra-jet and inter-jet pairs characterize the anisotropy

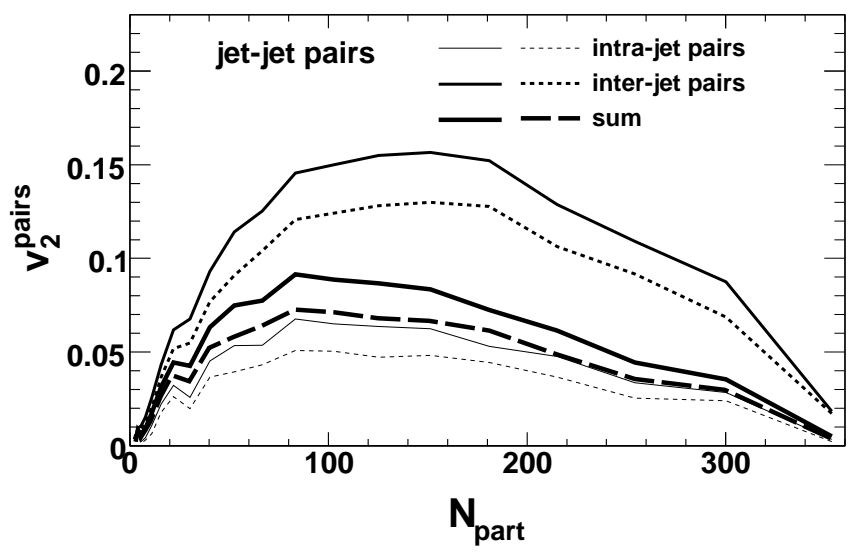

FIG. 3: The $v_{2}$ for jet-jet pairs calculated as $\left\langle\cos 2\left(\phi-\Psi_{R P}\right)\right\rangle$, where $\phi$ is the azimuthal angle of jets (solid lines) or fragmentation hadrons (dotted lines).

of the survival single- and di-jets, respectively. The former corresponds to the anisotropy of single jet modification factor $R_{A A}\left(\phi-\Psi_{R P}\right)$, i.e. $v_{2}^{\text {Pair }}=v_{2}^{R_{A A}}$, while the latter corresponds to the anisotropy of di-jet modification factor $J_{A A}=R_{\mathrm{AA}}\left(\phi-\Psi_{\mathrm{RP}}\right) I_{\mathrm{AA}}\left(\phi-\Psi_{R P}\right)$ [7], i.e $v_{2}^{\text {Pair }} \approx v_{2}^{R_{A A}}+v_{2}^{I_{A A}}$. The fact that $v_{2}^{I_{A A}}>v_{2}^{R_{A A}}$ (Fig. 3) implies a stronger suppression of $I_{A A}$ than $R_{A A}$ in our calculation. The 20-30\% difference between the solid and dashed lines is due to the smearing which results from the near- and away-side jet widths.

The $v_{2}$ values for the jet-medium and medium-medium pairs, calculated using the angle of the original jets (i.e., 
$\phi$ in Eq4 refer to jet angle) are much smaller than those for jet-jet pairs and even become negative. This is because more medium response hadrons are produced in the out-of-plane direction as a result of energy loss, i.e., there is more energy loss out-of-plane than in-plane. A similar path-length dependence has been predicted to lead to a negative $v_{2}$ for jet-conversion photons [19]. However, medium response hadrons are emitted at a large angle $(\mathrm{D}=1.1$ radian) relative to the original jet direction. Consequently, a much reduced $v_{2}$ is to be expected. In fact, when the angle of these hadrons are used in our analysis (i.e., $\phi$ in Eq4 refer to angle of one of the hadrons in the pair), the sign of $v_{2}$ is reversed, albeit with smaller magnitudes compared to those from jet fragmentation.

The anisotropy studied in this model originates purely from jet-quenching, other mechanisms could introduce new sources of $v_{2}$ for hadron pairs, which can change the values presented in Fig. 3. A measurement of the reaction plane dependence of the hadron pair yield (instead of the per-trigger yield), and the corresponding $v_{2}$ parameter, should be useful in helping us to constrain the underlying mechanisms of the medium response.

In summary, we have used a simple jet absorption model to investigate a possible influence of hadron pairs produced by quenched jets, on di-hadron angular correlations at intermediate $p_{T}$. The pair yield from quenched jets (medium-medium pair), often neglected in model calculations, is found to be significant when compared to jet-jet and jet-medium contributions. Using reasonable parameters for jet kinematics, we find that the inclusion of medium-medium pairs is sufficient to qualitatively reproduce the shape of experimental di-hadron

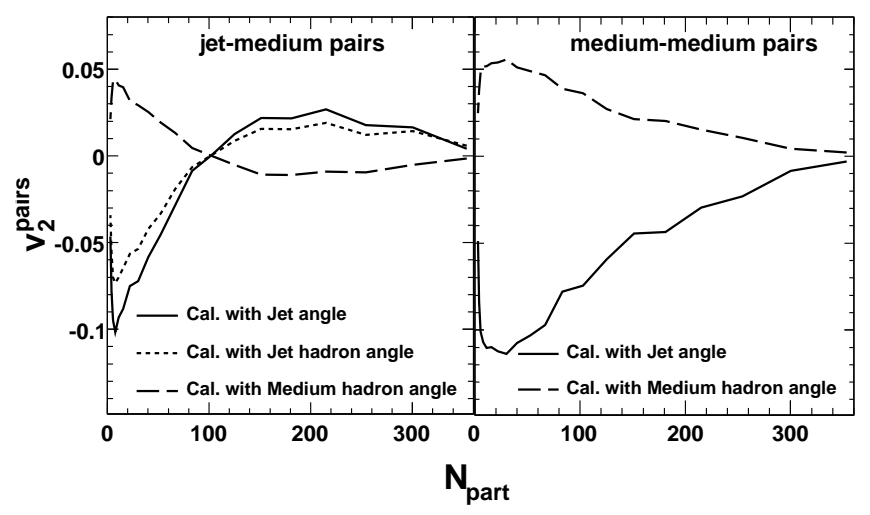

FIG. 4: The $v_{2}$ for jet-medium (left panel) and mediummedium (right panel) pairs calculated as $\left\langle\cos 2\left(\phi-\Psi_{R P}\right)\right\rangle$, where $\phi$ is the azimuthal angle of jets (solid lines) or fragmentation hadrons (dotted lines) or medium response hadrons (dashed lines).

$\Delta \phi$ distribution. Our simulation also suggest that these pairs may serve as a common source for the near-side ridge and away-side cone. This finding is consistent with experimental data [7, 8, 9] which show similar properties between the ridge and the cone (i.e., similar particle composition, spectra slope, etc). A distinct feature of medium-medium pairs is that their yield increase with path-length and the resulting anisotropy is very sensitive to their emission angle relative to that of the jet (also true for jet-medium pairs to a certain extent). The measurement of such anisotropies may provide important constraints for the medium response to jets.

This work is supported by the NSF under award no. PHY-0701487 and by the DOE under award no. 10112511-007968.
[1] W. A. Zajc, Nucl. Phys. A 805, 283 (2008)

[2] K. Adcox et al. Phys. Rev. Lett. 88, 022301 (2002)

[3] J. Adams et al. Phys. Rev. Lett. 97, 162301 (2006)

[4] A. Adare et al. Phys. Rev. C 77, 011901(R) (2008)

[5] R. J. Fries, S. A. Bass and B. Muller, Phys. Rev. Lett. 94, 122301 (2005)

[6] S. S. Adler et al. Phys. Rev. Lett. 97, 052301 (2006)

[7] A. Adare et al. Phys. Rev. C 78, 014901 (2008) and references therein.
[8] C. Alt et al., Phys. Rev. Lett. 101, 082301 (2008)

[9] A. Franz et al. J. Phys. G 35, (2008) 104002

[10] J. Adams et al. Phys. Rev. C 75, 034901 (2007)

[11] M. Daugherity [STAR Collaboration], J. Phys. G 35, (2008) 104090.

[12] I. Vitev, Phys. Lett. B 630, 78 (2005); V. Koch, A. Majumder and X. N. Wang, Phys. Rev. Lett. 96, 172302 (2006); N. Armesto, C. A. Salgado and U. A. Wiedemann, Phys. Rev. C 72, 064910 
(2005); H. Stoecker, Nucl. Phys. A 750, 121 (2005); J. Casalderrey-Solana, E. V. Shuryak and D. Teaney, hep-ph/0602183 C. B. Chiu and R. C. Hwa, Phys. Rev. C 74, 064909 (2006); A. D. Polosa and C. A. Salgado, Phys. Rev. C 75, 041901(R)(2007); A. Majumder, B. Muller and S. A. Bass, Phys. Rev. Lett. 99, 042301 (2007); C. Y. Wong, Phys. Rev. C 76, 054908 (2007); E. V. Shuryak, Phys. Rev. C 76, 047901 (2007).

[13] A. Drees, H. Feng and J. Jia, Phys. Rev. C 71, 034909
(2005)

[14] S. S. Adler et al. Phys. Rev. C 69, 034910 (2004)

[15] S. S. Adler et al. Phys. Rev. C 73, 054903 (2006)

[16] S. S. Adler et al. , Phys. Rev. C 69, 034909 (2004)

[17] J. Adams et al. , Phys. Rev. Lett. 93, 252301 (2004)

[18] J. Jia, J. Phys. G 35, (2008) 104033

[19] S. Turbide, C. Gale and R. J. Fries, Phys. Rev. Lett. 96, 032303 (2006) 